\title{
Los primeros arbitrajes internacionales en materia ambiental
}

\author{
The First International Environmental \\ Arbitrations
}
Les premiers arbitrages internationaux pour l'environnement

\section{Gabriel Ferrer Ortega*}

\begin{abstract}
SUMARIO: I. Introducción. II. Apuntes sobre la evolución del derecho ambiental internacional. III. Fur Seals Arbitration. IV. Trail Smelter Case. V. Arbitraje del lac Lanoux entre Francia y España. VI. La herencia de los primeros arbitrajes. VII. Bibliografía.
\end{abstract}

* “Centro Lindavista”, Centro de Investigación, Información y Apoyo a la Cultura, A. C., México; ORCID ID: https: / /orcid.org/0000-0003-0034-0104,gaboferrer@gmail.com.

Artículo recibido el 10 de marzo de 2020

Aprobado para publicación el 9 de octubre de 2020 
RESUMEN: Este trabajo discurre sobre las primeras controversias arbitrales internacionales en las que están presentes temas ambientales: Fur Seals Arbitration, United States and the United Kingdom (1893); Trail Smelter Arbitration, United States of America versus Canada (1938 y 1941), y Affaire du Lac Lanoux, Espagne versus France, (1957). Los dos primeros arbitrajes, resueltos antes de la creación de la Corte Internacional de Justicia, dan una idea de una primera concepción, un primer capítulo, del cambiante pensamiento humano hacia el entorno natural, sentando, junto al Affaire du Lac Lanoux, los primeros principios del derecho ambiental internacional.

Palabras clave: litigio internacional, litigio ambiental, derecho ambiental internacional, equidad intergeneracional, el que contamina paga, responsabilidad ambiental.

ABSTRACT: This work deals with the first international arbitration controversies in which environmental issues are present: Fur Seals Arbitration, United States and the United Kingdom (1893); Trail Smelter Arbitration, United States of America versus Canada (1938 and 1941); and Affaire du Lac Lanoux, Espagne versus France, (1957). The first two arbitrations, resolved before the creation of the International Court of Justice, give an idea of a first conception, a first chapter, of the changing human thought towards the natural environment, laying down, together with the Affaire du Lac Lanoux, the first principles of International Environmental Law. Key words: international litigation, environmental litigation, international environmental law, intergenerational equity, polluter pays, environmental responsibility.

RÉSUMÉ: Ce travail traite des premières controverses d'arbitrage international dans lesquelles des problèmes environnementaux sont présents: Fur Seals Arbitration, États-Unis et RoyaumeUni (1893); l'arbitrage Trail Smelter, États-Unis d'Amérique contre Canada (1938 et 1941); et Affaire du Lac Lanoux, Espagne contre France, (1957). Les deux premiers arbitrages, résolus avant la création de la Cour Internationale de Justice, donnent une idée d'une première conception, un premier chapitre, de l'évolution de la pensée humaine vers le milieu naturel, fixant avec l'Affaire du Lac Lanoux les premiers principes du droit international de l'environnement.

Mots-clés: litige international, litige environnemental, droit international de l'environnement, équité intergénérationnelle, pollueur-payeur, responsabilité environnementale. 


\section{INTRODUCCIÓN}

Historiar sobre las primeras controversias internacionales en materia ambiental sometidas a arbitraje es adentrarse en la conciencia de la afectación al medio ambiente por el hombre y buscar, en un plano todavía muy primitivo, las reparaciones a las afectaciones del entorno provocadas por la propia actividad humana. Los litigios internacionales fueron de vital importancia para resolver hechos controvertidos de una manera pacífica y legaron al futuro un acervo jurisprudencial que permite volver a ser utilizado en casos similares y proveer de guía normativa a las distintas áreas del derecho internacional, a la cual no es ajena la materia ambiental.

La selección de los casos Fur Seals Arbitration, United States and the United Kingdom (1893), Trail Smelter Arbitration, United States of America versus Canada (1938 y 1941) y Affaire du Lac Lanoux, Espagne versus France (1957) obedece al contenido eminentemente ambiental de ellos, sometido a un criterio cronológico, y a que fueron resueltos por tribunales arbitrales creados por las partes en contraposición a casos posteriores en materia ambiental que han sido presentados ante instancias jurídicas internacionales permanentes, como es el caso de controversias más modernas resueltas por la Corte Internacional de Justicia (CIJ) o el Tribunal Internacional del Derecho del Mar. No cabe duda que otros arbitrajes y litigios internacionales que no tienen un contenido ambiental han tenido repercusiones importantes en el derecho ambiental internacional, los cuales mencionaremos tangencialmente. Sin embargo, la intención es dejar en claro que desde hace muchos años algunas controversias ambientales son dirimibles y aportan a la evolución de la materia.

De esta manera, quedará en el estudio de estos casos desentrañar la importancia que tuvieron para resolver las cuestiones que se les presentaron y vincularles a la evolución del pensamiento sobre el entorno natural, así como la herencia que han dejado como un referente jurisprudencial e, incluso, en la formación de los primeros principios y reconocimiento de normas en materia ambiental. Por tanto, el estudio de casos no es meramente histórico, lo cual de por sí sería valioso, sino entrelazado a litigios subsecuentes y principios del derecho ambiental internacional. 


\section{APUNTES SOBRE LA EVOLUCiÓN DEL DERECHO}

AMBIENTAL INTERNACIONAL

Cierto es que en el pensamiento clásico no se encuentra una especial preocupación por el medio ambiente, aunque esto es entendible ante el fenómeno tan relativamente reciente de la trascendencia de presencia humana en nuestro planeta, claramente visible y medible en los últimos años. Todavía hace unas décadas campeaba la idea de que el actuar presente legaba un mejor futuro a las futuras generaciones, lo cual era confirmado por los avances científicos y tecnológicos y las grandes aportaciones culturales y sociales; idea cuestionada hoy en día por la clara huella de daño en nuestro entorno con tonos globales.

La reflexión moderna apunta que "El enorme incremento del potencial de la humanidad que tuvo lugar en el siglo XX explica porque aún los filósofos morales del pasado no prestaron atención de nuestra responsabilidad hacia la posteridad", de acuerdo con el filósofo alemán Jörg Tremmel; ${ }^{1}$ aunque esto no es necesariamente cierto del todo.

Tomás de Aquino señalaba que "compete al hombre, respecto de los bienes exteriores, el uso de los mismos; y en cuanto a esto no debe tener el hombre las cosas exteriores como propias, sino como comunes, de modo que fácilmente dé participación de éstas en las necesidades de los demás”, 2 lo que significa una restricción al abuso o al daño.

Locke, por su parte, afirmaba que los bienes comunes de la Tierra (y animales inferiores) son originariamente comunes para todos los hombres, pueden ser removidos de su estado de naturaleza y apropiados, junto con lo que se añada a estos bienes por el trabajo del hombre, aunque también impone un límite importante, siempre y cuando existan "suficientes bienes y tan buenos en común para otros". ${ }^{3}$

1 Tremmel, Jörg, A Theory of Intergenerational Justice, Londres, Earthscan, 2009, p. 3.

2 Summa Theologiae, 2a2ae, 66, 2. Literalmente apuntaba el doctor angélico: "Aliud vero quod competit homini circa res exteriores est usus ipsarum. Et quantum ad hoc non debet homo habere res exteriores ut proprias, sed ut communes, ut scilicet de facili aliquis ea communicet in necessitates aliorum”. En español puede consultarse Aquino, Tomás, Suma de Teología, parte II-II (a), trad. de Lorenzo Jiménez Patón, Madrid, Biblioteca de Autores Cristianos, 1990, pp. 544 y 545.

3 Locke, John, Two Treatises of Government, Londres, A. Millar et al., 1764, p. 216, disponible en: https://oll.libertyfund.org/titles/locke-the-two-treatises-of-civil-government-hollis-ed. La 
En el otro extremo del pensamiento, Karl Marx apuntaba que ni siquiera "todas las sociedades contemporáneas reunidas, son propietarias de la tierra", y agrega el economista y filósofo alemán que "Sólo son sus poseedoras, sus usufructuarias, y deben legarla mejorada, como boni patres familias, a las generaciones venideras". ${ }^{4}$

Esta pequeña muestra, en la escolástica, el pensamiento liberal y el marxista, nos deja ver que de siempre ha existido una noción de limitación al disfrute de los bienes comunes y de nuestro propio planeta. Quedaría la tarea de ligar el pensamiento al fenómeno jurídico, limitar el uso de los bienes comunes o construir el andamiaje jurídico para la construcción de un usufructo planetario.

Ahora bien, en el plano legal, los primeros cuerpos normativos que tienen un atisbo de protección ambiental apenas aparecen en el siglo XIX. ${ }^{5}$ Por lo que toca al ámbito doméstico, es común la idea de que el primer ordenamiento en los Estados Unidos (EUA) fue la Refuse Act de 1899, ${ }^{6}$ creada con el objeto de impedir el vertimiento de desperdicios en aguas navegables, aunque su intención original estaba más enfocada en prohibir obstrucciones a la navegación. Puede, sin embargo, señalarse con toda propiedad que la primera disposición ambiental en los EUA de la que se tiene registro fue la Yosemite Grant, firmada por Abraham Lincoln el 30 de junio de 1864, ordenamiento mediante el cual la Federación cedería a California el valle de Yosemite bajo la condición de que este fuera para uso público y recreativo y que cualquier ingreso fuera utilizado para la conservación, mejoramiento y protección del espacio. ${ }^{7}$ Este decreto fue, a su vez, un antecedente para la creación del Parque Nacional de Yellowstone, en 1872 .

discusión sobre la propiedad se encuentra en el capítulo $\mathrm{V}$ del libro 2, y las citas en las secciones 26 y 27. En español puede consultarse Locke, John, Segundo tratado sobre el gobierno civil, trad. de Carlos Mellizo, Madrid, Tecnos, 2010, p. 33 y 34.

4 Marx, Karl. El capital, Biblioteca del Pensamiento Socialista, México, Siglo XXI, 2007, t. III, vol. 8, sección sexta, capítulo XLVI, p. 987.

5 Para una apreciación de la historia del derecho ambiental internacional puede consultarse, inter alia, el capítulo dedicado a ello en la obra Bodansky, Daniel, The Art and Craft of International Environmental Law, Cambridge, Harvard University Press, 2010, pp. 19-35.

6 En este sentido, véase Newton E., David, Environmental Justice: A Reference Handbook, 2 a. ed., California, ABC-CLIO, LLC, 2009, p. 113.

7 U. S. C., title 16, sec. 48. 
En cuanto toca al ámbito bilateral, los primeros tratados que tocan tangencialmente algunos aspectos sobre el medio ambiente tratan sobre el uso de ríos fronterizos, acceso al mar, pesquerías y caza. No es dable en este breve estudio detenernos en las particularidades de cada tratado, pero podemos adelantar que en dichos instrumentos las finalidades apuntan más hacia la explotación y poco a la racionalidad de recursos.

En la arena multilateral es señalado como el primero de los acuerdos multilaterales ambientales (MEAs, por sus siglas en inglés) el Tratado para la Regulación del Flujo de las Aguas del Lago de Constanza, concertado por los Estados ribereños en 1857, con miras a evitar los efectos perjudiciales de niveles excesivamente altos de agua en dicho lago, para lo cual se prohibían construcciones como molinos de agua. ${ }^{8}$

Para ilustrar con mayor claridad la sustancia de los primeros MEAs, conviene adentrarse en el texto de alguno de ellos. A guisa de ejemplo, en 1900 se firmaría, en Londres, la Convención para la Preservación de los Animales Silvestres, Pájaros y Peces de África, celebrada entre potencias coloniales europeas: Alemania, España, Francia, Italia, Portugal, Reino Unido y el atroz Estado Libre de El Congo. Aunque intenta crear un régimen de protección para la vida silvestre en el norte de África, entre las finalidades del instrumento sobresalía la conservación de animales para ser usados como trofeos de caza y por el uso de su pieles y productos; más todavía, llegó a incluir un grupo de animales que consideraba de interés reducir su número, como los cocodrilos, serpientes venenosas y pitones, así como leones, leopardos, hienas, perros salvajes, babuinos y "otros monos dañinos", algunas aves de rapiña y nutrias. ${ }^{9}$

Solamente dos años más tarde se concertó, en París, la Convención para la Protección de Aves Útiles para la Agricultura, resultado de un proceso que venía gestándose décadas atrás para establecer un régimen de prohibición de la caza, captura de nidos y cuidado de sitios de reproducción de ciertas aves, principalmente insectívoras, que favorecían la producción agrícola. Suerte que no se destinaba a ciertas aves depredadoras y a un grupo de roedores, ignorándose en esa época los servicios ecosistémicos que prestan

8 Disponible en: http://iea.uoregon.edu/treaty-text/1857-regulationflowwaterlakeconstancedetxt.

9 Para la historia de esta convención y las subsecuentes en la materia, véase Lyster, Simon, International Wildlife Law: An Analysis of International Treaties Concerned with the Conservation of Wildlife, Cambridge University Press, 1985, pp. 112-128. El texto del instrumento está disponible en: http: / / iea.uoregon.edu/pages/view_treaty.php?t=1900-PreservationWildAnimalsBirdsF ishAfrica.EN.txt\&par=view_treaty_html. 
todas las especies. ${ }^{10}$ Muy lejos están todavía estas disposiciones a nuestro entendimiento actual; puede señalarse que las concepciones del moderno derecho internacional dieron un gran paso con la Conferencia de Naciones Unidas sobre el Medio Humano de Estocolmo, de 1972, ${ }^{11}$ tomando en cuenta que el pensamiento debe evolucionar ante el reto ambiental actual.

La concepción señalada fue evolucionando en los arbitrajes internacionales, como daremos cuenta de ello, sentando las bases de un incipiente derecho ambiental internacional. Habrá de guiar este estudio la búsqueda de los primeros principios en la materia.

\section{FUR SEALS ARBITRATION}

Con una gran anticipación, la primera señalización, el primer argumento verdaderamente jurídico en favor de la conservación de la naturaleza y la preservación del hábitat humano para las futuras generaciones, expuesto en términos sólidos, fue expresado en 1892 por el agente estadounidense en el arbitraje sobre las focas peleteras del mar de Bering, el Bering Sea Fur Seal Fisheries Arbitration (Great Britain vs. United States). El argumento ha quedado enterrado en un injusto olvido, y por el valor intrínseco de los alegatos iusnaturalistas en favor de la conservación de los recursos naturales es dable hacer un recordatorio de sus principales líneas de argumentación en el contexto del arbitraje. Pero antes de ello, es necesario hablar de la problemática que dio origen a la controversia.

\section{Antecedentes}

En 1867 los EUA compraron el territorio de Alaska e islas adyacentes a Rusia, dividiendo en una línea media la zona marina entre ambas potencias. En esta división quedaron las islas Pribilof del lado estadounidense, área

10 Disponible en: http://www.ecolex.org/details/treaty/convention-for-the-protection-of-birdsuseful-to-agriculture-tre-000067/.

11 En este sentido, véase Brown Weiss, Edith, "The Evolution of International Environmental Law", 54 JapaneseY. B. Intl. L., pp. 1-27, 2011. 
de reproducción de la foca peletera de Alaska (Callorhinus ursinus), especie cuya población descendió de 2.5 millones de ejemplares calculados en 1786 a 130,000 en 1910, diezmo provocado por la brutal cacería a la que fue sujeta; pesca pelágica que afectaba en particular a hembras preñadas en el transcurso de su migración. ${ }^{12}$

Ante la situación señalada, el Congreso de EUA emitió ordenamientos entre 1868 y 1873, prohibiendo, salvo casos específicos, la matanza de focas peleteras en las islas Pribilof y en "las aguas adyacentes a ella", definiendo el secretario del Tesoro, en 1881, que todas las aguas al este de la línea de límite se considera que están dentro de las aguas del territorio de Alaska, lo que fue comunicado en 1886 por el secretario de Hacienda a todas las aduanas.

En el mismo año de 1886, tres botes de foqueros canadienses (Carolena, Onward y Thornton) fueron capturados a 60 millas de tierra firme por el Servicio de Impuestos de los Estados Unidos (hoy Guardia Costera). Aunque hubo negociaciones diplomáticas para que fueran liberadas las embarcaciones, un juez de distrito resolvió que la captura de focas fue realizada dentro de territorio estadounidense, lo que permitió el sustento para que otros botes foqueros corrieran la misma suerte. ${ }^{13}$

Debe recordarse que, por aquellos años, los asuntos exteriores de Canadá eran atendidos por el Imperio británico, que repudió el reclamo estadounidense y se unió a Rusia para entablar negociaciones con EUA para buscar un acuerdo que le permitiera tener acceso a este recurso pelágico.

Las negociaciones no llegaron a buen puerto, fue entonces cuando el primer ministro británico, lord Salisbury, propuso el arbitraje en 1890. Una vez consentida la propuesta por el secretario de Estado, James Gillespie Blaine, ambas partes acordaron los puntos que habrían de ser referidos al cuerpo arbitral en un compromiso firmado el 29 de febrero de 1892, cuerpo que tendría que resolver cinco cuestiones, de las cuales prestaremos particular atención a la última de ellas:

1) ¿Qué jurisdicción exclusiva tenía Rusia sobre el mar de Bering y sus recursos, antes y al momento de ceder Alaska a los EUA?

12 Older, Owen S., Conservación de recursos naturales, 2a. ed., trad. de Ambrosio González Cortés, México, Pax, 2000, p. 507.

13 Para más detalles véase Brown, J. Stanley, "Fur Seals and the Bering Sea Arbitration", Journal of the American Geographical Society of New York, vol. 26, núm. 1, 1894, pp. 326-372. 
2) ¿Hasta qué punto los reclamos de jurisdicción sobre la caza de focas habrían sido reconocidos por la Gran Bretaña?

3) ¿Estaba incluido el mar de Bering en la frase "océano Pacífico" utilizada en el tratado de 1825 entre Gran Bretaña y Rusia? ¿Qué derechos pudo haber ejercido Rusia en el mar de Bering después de dicho tratado?

4) ¿Pasaron intactos todos los derechos de jurisdicción y sobre la caza de focas en el mar de Bering en el tratado entre los EUA y Rusia de 1867 ?

5) ¿Tienen los EUA un derecho de protección o propiedad sobre las focas peleteras que frecuentan el mar de Bering cuando aquellos mamíferos se encuentran fuera del límite de las tres millas?

\section{Argumentación estadounidense}

El agente de los EUA, James Coolidge Carter, como premisa, buscó colocar a su país como soberano de las focas peleteras, casi 100 años antes de que se cristalizara el concepto de Zona Económica Exclusiva. Sobre la cuestión de conservación, Carter afirmaba que la propiedad no es absoluta, pues la noción de que el titular puede abusar de ella e incluso destruirla es desacertada. Pensar que la limitación del propietario es solamente moral o religiosa, que es responsable solamente ante su creador y no ante leyes humanas es igualmente equívoco, añadiría Carter. ${ }^{14} \mathrm{Y}$ en dos puntos resumiría Carter esta postura: i) ningún individuo o nación tiene un derecho absoluto sobre la propiedad que posee, su título está limitado por un fideicomiso en beneficio de la humanidad y ii) el título es aún más limitado. Las cosas mismas no le son dadas, sólo el usufructo o las creces. No es más que el custodio de las acciones o de la cosa principal, detentándola en fideicomiso para las generaciones presentes y futuras del hombre. ${ }^{15}$ No habría sido la exposición de Carter un argumento lírico, el agente estadounidense sostendría sus afirmaciones en la aserción de Puffendorf: "Dios dio el mundo, no a un hombre u a otro, sino a toda la

14 Fur Seal Arbitration, Argument of the United States Before the Tribunal of Arbitration Convened at Paris Under the Provisions of the Treaty between the United States of America and Great Britain, Concluded February 29, 1892, Washington, Govt. print. Off, 1893, pp. 518 y 519.

15 Ibidem, p. 519. 
humanidad en general", ${ }^{16}$ y en la aseveración de Locke: "Dios dio el mundo a los hombres en común". ${ }^{17}$

Vale la pena profundizar en el pensamiento de Puffendorf. En el mismo libro De iure naturae et Gentium, citado por Carter, también se apunta que la razón lleva al hombre a usar sus posesiones con moderación para su propio servicio y el de sus dependientes. Este principio no impide que se preocupe por el futuro, siempre y cuando no sea por envidia o avaricia que prevenga a otros de su aprovechamiento. No son accidentales las aseveraciones de Puffendorf, fueron extraídas del capítulo "Del origen del dominio o la propiedad", las cuales, sin ser una premonición de los problemas que la humanidad enfrenta por el deterioro ambiental, constituyen principios naturales aplicables a todos los tiempos y circunstancias.

En continuación al argumento, Carter retoma la idea central de Locke: "Dios dio la tierra a los hombres en común", y le añade como ingrediente que una nación sería fiduciaria de los frutos que fueran excedentes a sus necesidades. Carter también discute la posibilidad del descuido a este deber: "destruir la fuente de donde fluya cualquier bendición humana no es simplemente un error, es un crimen.Y el mal no está limitado por las fronteras de las naciones, es infligido a aquellos a quienes la bendición sería útil dondequiera moren". ${ }^{18}$ Más allá, Carter prevé un mecanismo; en estos casos el agente estadounidense afirma que habría una acción de reparación. ${ }^{19}$

Asimismo, Carter intentaría construir lo que hoy es reconocido como el principio de equidad intergeneracional, ${ }^{20}$ lo que pudo haber constituido

16 Puffendorf, Samuel, Of the Law of Nature and Nations, Londres, J. Walthoe et al., 1729, pp. 383 y 384, disponible en: https://openlibrary.org/books/OL6563001M/Of_the_law_of_na ture_and_nations.

17 Locke, John, Two Treatises of Government, cit., p. 216.

18 Fur Seal Arbitration, Argument of the United States..., cit. pp. 63 y 64.

19 Ibidem, p. 64.

20 Para un estudio profundo de este principio véase Ferrer Ortega, Luis Gabriel, Los derechos de las futuras generaciones desde la perspectiva del derecho internacional: el principio de equidad intergeneracional, México, UNAM, Instituto de Investigaciones Jurídicas, 2014, disponible en: https: / / biblio.juridicas.unam. $\mathrm{mx} / \mathrm{bjv} /$ detalle-libro/3635-los-derechos-de-las-futuras-generaciones-desde-la-perspectiva-del-derecho-internacional-el-principio-de-equidad-intergeneracional; Ferrer Ortega, Luis Gabriel y Ferrer Ortega, Jesús Guillermo, "El problema de la fundamentación filosófica de los derechos de las generaciones futuras", Anuario Mexicano de Derecho Internacional, México, vol. VIII, 2008. 
un verdadero salto cuántico si hubiera tenido resonancia su argumentación. Carter propondría los siguientes principios:

- La naturaleza no pertenece a una sola nación, pertenece a toda la humanidad en su conjunto.

- En la definición de humanidad, todas las generaciones, futuras y presentas, están incluidas.

- La tierra está diseñada para ser la morada permanente del hombre a través de generaciones interminables.

- Cada generación tiene solamente el derecho a usar equitativamente su herencia.

- Es contrario al derecho natural comprometer a la siguiente generación con desechos.

- Cada generación debe transmitir su herencia por lo menos en la misma condición que la recibió.

- La generación presente no puede consumir o destruir el incremento de los productos de la tierra, y menos los propios recursos, dejando una provisión inadecuada para que se multipliquen esos recursos.

Este punto es un adelanto a la fundación de una teoría de equidad intergeneracional como la plantearía 100 años después Edith Brown Weiss, en la obra In Fairness to Future Generations, bajo tres principios básicos:

1) Conservación de opciones. Cada generación deberá conservar la diversidad de la base de sus recursos naturales y culturales con el fin de no coartar indebidamente las opciones de generaciones futuras para solucionar sus problemas y satisfacer sus propios valores. Igualmente tendrá el derecho a gozar una diversidad comparable a la que disfrutó la generación previa.

2) Conservación de calidad. Cada generación deberá mantener la calidad de la tierra para que pueda ser entregada en una condición que no sea inferior de lo que la recibió.

3) Conservación de acceso. Cada generación deberá otorgar a sus miembros derechos equitativos para que puedan acceder al legado de la generación anterior y deberá conservar este acceso a las generaciones posteriores. ${ }^{21}$

21 Brown Weiss, Edith, In Fairness to Future Generations. International Law, Common Patrimony and Intergenerational Equity, Tokio, United Nations University Press, 1989, pp. 34-44 
Como puede observarse, existe un gran paralelismo no sólo en la enunciación de los principios básicos, sino también en la propia cimentación. Para Brown Weiss, la equidad intergeneracional se enfoca "en la relación inherente que cada generación tiene con otras generaciones, pasadas y futuras, en el uso del patrimonio común de recursos naturales y culturales del planeta". Entendida la generación presente como "custodia del planeta", ciertas obligaciones se pueden transformar en normas ejecutables "[c] omo beneficiarios del legado de las generaciones pasadas, hemos heredado ciertos derechos a gozar los frutos de este legado, tal como lo harán las futuras generaciones". ${ }^{22}$

Regresando al litigio, Carter, anticipando cualquier argumentación positivista, intentó señalar que sus razonamientos eran autodemostrables, y que por ello ningún pensador reparó en justificarlas, bastando la propia mención para tenerlas por asentadas. El litigante estadounidense también argumentaba que el hombre primitivo, con menores necesidades, jamás pondría en peligro los recursos naturales ni tendría que aprovisionarse para el futuro; sin embargo, con el avance de la civilización, el incremento poblacional y la multiplicación de las necesidades podría sobrevenir un peligro de sobreconsumo, al que le acompañaría el desarrollo de una visión prudencial que buscaría evitar el peligro. ${ }^{23}$ Ojalá y hubieran sido desacertadas las aseveraciones del agente estadounidense, pero hoy en día es constatable que se actualizan sus hipótesis.

\section{Réplica británica}

No sería extraño que la contraparte inglesa contrarrestara los argumentos estadounidenses en la tradicional concepción del término de la sobera-

$22 \mathrm{Ibidem}, \mathrm{p} .21$. "Intergenerational equity arises in the context of fairness among all generations. The theory of intergenerational equity proposed here focuses on the inherent relationship that each generation has to other generations, past and future, in using the common patrimony of natural and cultural resources of our planet. The starting proposition is that each generation is both a custodian and a user of our common natural and cultural patrimony. As custodians of this planet, we have certain obligations to future generations which we can transform into legally enforceable norms. Our ancestors had such obligations to us. As beneficiaries of the legacy of past generations, we inherit certain rights to enjoy the fruits of this legacy, as do future generations. We may view these as intergenerational planetary obligations and planetary rights".

23 Ibidem, p. 64, "But with the advance of civilization, the increase in population, and the multiplication of wants, a peril of overconsumption arises, and along with it a development of that prudential wisdom which seeks to avert the danger". 
nía al terminarse la franja de las tres millas marinas y replicara que los EUA se refugiaban en una pretendida protección de lo que no les corresponde en propiedad de forma tan novedosa: "Que sus partidarios confiesan con candor que no puede descansar en ningún precedente sino que un precedente debe ser establecido por el derecho internacional para satisfacer las exigencias del caso". ${ }^{24}$ No sin cierta sorna, el argumento inglés echaría por tierra la tesis iusnaturalista de Carter, señalando que, en cuanto a la referencia a las "leyes de la historia natural", no se conoce el código de tales leyes, y en lo que toca a los "intereses comunes de la humanidad", éstos deben ser probados por y con los principios legales. Se remataría esta idea, bajo una tesis voluntarista y positivista, la cual predomina con escasas excepciones en nuestros días: en el derecho internacional no pueden crearse espontáneamente principios novedosos, el consentimiento de los Estados, esencial para la admisión de un nuevo precedente, no puede ser presumido. ${ }^{25}$

La visión inglesa sobre la conservación de la naturaleza era muy lejana a la visión del litigante estadounidense, tal como lo refleja la Convención para la Preservación de los Animales Silvestres, Pájaros y Peces de África firmada en 1900 y de la que hemos comentado que tenía miras diferentes a lo que podría ser un MEA actual.

\section{Laudo arbitral}

La respuesta y la reacción de la parte inglesa es conocida, lo que no puede saberse a ciencia cierta es el efecto que causó el argumento estadounidense en los arbitradores. En cierto sentido, la visión anticipatoria de Carter sería el equivalente a un texto de Julio Verne ${ }^{26}$ en aquellos años, y lamentablemente los considerandos del laudo son escuetos, por lo que son

24 Bering Sea Tribunal of Arbitration, Proceedings of the Tribunal of Arbitration Convened in Paris Under the Treaty between the United States of America and Great Britain, Concluded at Washington, February 29, 1892, for the Determination of Questions between the Two Governments Concerning the Jurisdictional Rights of the United States in the Waters of Bering Sea, Argument of Her Majesty's Government, Washington, G. P. O., 1895, vol. X, p. 9.

25 Ibidem, p. 34.

26 Curiosamente, Verne falleció el mismo año que el litigante estadounidense (1905) y habría nacido unos meses después de Carter (febrero de 1828 y octubre de 1827, respectivamente). 
inciertas las reflexiones que llevaron a los togados a pronunciarlo el 15 de agosto de 1893.

A pesar de ello, y aunado a que prevalecería el Reino Unido en todos los puntos $^{27}$ sobre los EUA, incluido el quinto, se resolvería afirmando que este último país no tenía ningún derecho a proteger a las focas peleteras cuando aquellas se encuentran fuera del límite reconocido de las tres millas. He aquí un antecedente valioso sobre el derecho de protección a los recursos naturales en zonas marinas, al cual se le uniría más tarde el laudo arbitral en el caso de las Pesquerías en la Costa del Atlántico Norte, ocasión en que los árbitros señalarían que el Estado costero no tendría el derecho sino la obligación de proteger y preservar las pesquerías en el mar bajo su jurisdicción, ${ }^{28}$ lo cual vemos hoy en día reflejado en el artículo 193 de la Convención de las Naciones Unidas sobre el Derecho del Mar (Convemar).

Pues bien, pareciera que algún efecto habría causado el argumento de Carter en el ánimo de los arbitradores, ya que más adelante se resolvería que podrían establecerse ciertas regulaciones con el concierto de ambos países. En esto, debe reconocerse que fue un gran acierto la estrategia estadounidense al haber convencido a la parte británica de facultar al tribunal para proponer regulaciones para la protección y preservación de la especie. Fue una batalla ganada en este punto desde antes de haber comenzado; los arbitradores, aunque reconocieron que los EUA no tendrían ningún derecho a proteger a las focas peleteras más allá del límite de las tres millas, se vieron obligados a redactar un proyecto de regulaciones para las partes.

En concreto, el tribunal redactó un articulado ex profeso entre cuyas propuestas se incluyeron: una zona de protección de 60 millas alrededor de las islas Pribilov, prohibir la captura durante la época del año comprendida entre el 1o. de mayo y el 31 de julio, ${ }^{29}$ un régimen de licencias para las

27 Incluida una reparación. La cuantificación de daños fue decidida por una comisión mixta establecida entre ambas partes en 1896, la que otorgaría a Gran Bretaña la suma de $\$ 473,151.26$, que equivaldría a más de 10 millones de dólares si se ajustara la cantidad en nuestros días.

28 Permanent Court of Arbitration, The North Atlantic Coast Fisheries Case (Great Britain, United States), Award of 7 September 1910, Reports of International Arbitral Awards, p. 187.

29 Bering Sea Tribunal of Arbitration, Award of the Tribunal of Arbitration Constituted Under Article I of the Treaty Concluded at Washington on the 29th February, 1892, between Her Britannic Majesty and the United States of America, Londres, Her Majesty Stationery Office-Harrison and Sons, 1893, p. 10. Expresaría, literalmente: "Los dos Gobiernos deberán prohibir a sus ciudadanos y súbditos respectivamente la muerte, captura y persecución de focas peleteras en Alta 
embarcaciones involucradas en la caza de la foca peletera, conteo de individuos capturados, intercambio de información sobre operaciones de pesca y captura humanitaria (prohibición de redes, armas de fuego y explosivos, control de aptitudes y destreza de pescadores y, como remate, protección a la pesca tradicional de grupos étnicos).

Considerando que la resolución data de más de un siglo de existencia, no dejaba de ser novedoso el contenido de este proyecto de articulado para el terreno internacional, y para cualquier otro, podríamos añadir. Aunque no se adaptó la propuesta arbitral, sin duda contribuiría a la firma de un tratado convenido entre el Reino Unido, los EUA, Japón y Rusia con miras a la protección de la foca peletera. ${ }^{30}$

Lo que más hemos querido destacar de este arbitraje es la visión del jurista Carter, muy adelantada a su tiempo y expresada en términos similares a la teoría que propondría casi 100 años después Edith Brown Weiss para las futuras generaciones, así como la primera propuesta de un régimen de protección fuera de los límites de la jurisdicción nacional.

Ahora bien, ¿cuál es el valor del argumento estadounidense dentro del caso en comento? Para el derecho internacional es comúnmente aceptado que la ratio decidendi de una resolución es la parte que tiene mayor valor normativo; ${ }^{31}$ por consiguiente, el argumento de una de las partes visto de manera aislada no tendría ningún valor. Sin embargo, las proposiciones de una de las partes pueden servir de guía para entender las razones por las cuales un juzgador se inclina en un sentido u otro. Por no estar expreso en las razones que le llevaron a proponer las regulaciones para las partes, queda en el terreno de la conjetura que el argumento de Carter pudo haber influido en la pluma de los arbitradores. A pesar de ello, no sería la última mención de lo que hoy se conoce como el principio de equidad in-

Mar, de cualquier forma que sea, durante el periodo annual comprendido entre el primero de mayo al 31 de julio, en el Oceano Pacífico, inluyendo el Mar de Bering (the two Governments shall forbid their citizens and subjects respectively to kill, capture, or pursue, in any manner whatever, during the season extending, each year, from the 1st May to the 31 st July, both inclusive, the fur-seals on the high sea, in the part of the Pacific Ocean, inclusive of the Behring Sea)".

30 North Pacific Fur Seal Convention of 1911, formalmente titulada: Convention between the United States and Other Powers Providing for the Preservation and Protection of Fur Seals.

31 Jennings, (Sir) Robert Y., "Judicial Reasoning at an International Court", en Ress, G. (ed.), Vorträge Reden und Berichte aus dem Europa-Institut, Saarbrücken, núm. 236, 1991, pp. 6 y 7. 
tergeneracional; correspondería al juez Weeramentry volver a retomarlo con fuerza en su opinión individual en el caso Gabč́kovo-Nagymaros Project (Hungary/Slovakia) ante la CIJ, ${ }^{32}$ después de un ayuno judicial de casi 100 años de la necesaria preocupación por las futuras generaciones $\mathrm{y}$, posteriormente, en su opinión individual en el caso Whaling in the Antarctic (Australia vs. Japan: New Zealand intervening), ${ }^{33}$ obra jurisprudencial que, junto con otras fuentes de derecho internacional, lleva a la solidificación del principio de equidad intergeneracional. He ahí el legado de Carter.

\section{TRAIL SMELTER CASE}

\section{Antecedentes}

El caso Trail Smelter tuvo su origen en las reclamaciones de EUA a Canadá por las emisiones de una fundidora de zinc y plomo ubicada en el poblado de Trail, British Columbia, las cuales causaron daño a un número de granjas ubicadas en el estado de Washington.

Como un dato curioso que arroja luz sobre la particularidad del caso y la trascendencia de la fundidora en la vida cotidiana, el muy famoso equipo local de hockey sobre hielo, deporte nacional canadiense, es conocido como los "tragahumos" (smokeaters). La conexión entre la economía y vida cotidiana del poblado canadiense se ilustra con su antiguo lema: "Entre más grueso el humo que asciende de la colina de la fundidora, mayor es la prosperidad”. Por supuesto, los estudios sobre la fundidora de Trail aclaran que la prosperidad llevó aparejada la afectación al medio ambiente en los alrededores

32 "La noción de no causar daño a otros y de ahí sic utere tuo ut alienum non laedas fue una noción central en el budismo. Esto se traduce fluidamente en las posturas ambientales. «Alienum» en este contexto puede ser extendido por el budismo a las futuras generaciones también, y a otros elementos componentes del orden natural más allá del hombre mismo, ya que el concepto budista del deber tiene un largo alcance”, entre otros pasajes: GabĆ́íkovoNagymaros Project (Hungary/Slovakia), ICJ Reports, Judgment of 25 September 1997, Separate Opinion of Vice-President Weeramantry, p. 102.

33 Whaling in the Antarctic (Australia v. Japan: New Zealand Intervening), ICJ Reports, Judgment of 31 March 2014, Separate opinion of Judge Cançado Trindade, pp. 41-47. 
de la planta, describiendo las transformaciones de bosques de coníferas y campos en verdaderos "paisajes lunares". ${ }^{34}$

Pues bien, la fundidora se instaló en 1896, a 11 millas de la frontera con los EUA siguiendo el cauce del río Columbia, y fue adquirida en 1906 por la minera canadiense Consolidated Mining and Smelting Company. A partir de 1925 aumentó su producción, lo que resultó en mayores emisiones de bióxido de azufre, que causaron daños en el estado de Washington entre 1925 y 1931. Dos años después, el cónsul general estadounidense en Ottawa presentaría la cuestión al gobierno de Canadá, y en el mismo año los EUA propondrían que la problemática fuera remitida a la Comisión Internacional Conjunta, establecida entre ambos países y cuya función sería llevar a cabo una investigación y presentar un informe. ${ }^{35}$

Para 1931 la Comisión Internacional Conjunta presentaría su informe, cuantificando los daños al 1o. de agosto en 350 mil dólares y recomendando que se tomaran medidas para que la fundidora redujera sus emisiones.

No fue suficiente la labor de la Comisión. La parte canadiense se había dado por satisfecha con el informe y las recomendaciones, no así la parte estadounidense, a pesar de que la fundidora de Trail había iniciado labores para reducir a un tercio la emisión de azufres.

\section{El planteamiento arbitral}

Ante esta disensión, las partes habrían de recurrir al arbitraje. Mediante un compromiso arbitral firmado en 1935 se estableció un tribunal, cuyo artículo III le encomendaría resolver, en resumidas cuentas, los siguientes puntos: i) si existió daño después del 1o. de enero de 1932 y, en su caso, cuál sería la indemnización; ii) si la fundidora debería abstenerse de causar

34 Cfr. Allum, James R., “«An Outcrop of Hell»: History, Environment, and the Politics of the Trail Smelter Dispute”, en Bratspies, Rebecca M. y Miller, Russell (eds.), Transboundary Harm in International Law Lessons from the Trail Smelter Arbitration, Cambridge University Press, 2006, pp. 13-36.

35 Esa sería la función para la Comisión, sin facultades para emitir resoluciones, de acuerdo con el artículo 9o. del Boundary Waters Treaty, firmado por ambos países en 1909. Treaty between Great Britain and the United States relating to Boundary Waters and Questions Arising between the United States and Canada, 11 Jan. 1909, 36 Stat. 2448. 
daño y hasta qué punto; iii) las medidas o régimen que debía adoptarse, de ser el caso, y iv) la indemnización o compensación que debería pagarse. ${ }^{36}$

Para responder a los planteamientos presentados, los árbitros debían aplicar "el derecho y la práctica seguida [...] en los Estados Unidos de América, así como el derecho y la práctica internacional”, ${ }^{37}$ cuestión que no era menor, pues si bien es cierto que se contaba con algunos precedentes judiciales en ese país, en el terreno ambiental internacional no habría pronunciamientos arbitrales o convencionales. El panorama podría ser inclusive sombrío de considerarse que los propios EUA adoptaron por algún tiempo la llamada Doctrina Harmon, que prescribía, en términos llanos, que ese país podría llevar a cabo cualquier actividad en el río Colorado sin importar las afectaciones que le causaran a su vecino: México. ${ }^{38}$

Por otro lado, puede señalarse que el laudo, en buena medida, fundó su decisión en el espíritu del famoso caso Alabama, que menciona, en diversas ocasiones y de forma contundente, que Gran Bretaña tenía el deber de prevenir la construcción de un buque destinado a causar presa de embarcaciones de la Unión durante la Guerra Civil estadounidense. ${ }^{39}$

\section{El Laudo arbitral}

Si bien a la argumentación podrían dedicarse numerosas páginas, habremos de obviarla y enfocar los esfuerzos al contenido del fallo y a la evolución de sus ideas centrales. Podría hablarse de dos laudos o, con mayor propiedad, de un laudo compuesto por dos textos complementarios emitidos en diferentes fechas. En efecto, el 16 de abril de 1938, el tribunal publicó su "decisión final” a la cuestión 1, decisiones temporales a las cuestiones 2 y 3, decretando un régimen para las partes, y la decisión definitiva habría de publicarse el 11 de marzo de 1941.

36 Convention Between the United States of America and the Dominion of Canada Relative to the Establishment of a Tribunal to Decide Questions of Indemnity and Future Regime Arising from the Operation of Smelter at Trail, British Columbia, 49 Stat. 3245.

37 Ibidem, artículo IV.

3821 Op.Att'y Gen. 274 (1895).

39 Alabama Claims Arbitration, 1872, Moore, 1 International Arbitration 495 y 29 RIAA, $125-134$ 
La resolución de los árbitros asentó dos principios fundamentales en el derecho ambiental internacional: uno de ellos, que "los Estados tienen el deber de prevenir el daño transfronterizo", y dos, como consecuencia del primero, "el que contamina paga", resultado ineludible de la ecuación de atribución del daño y responsabilidad. Es por ello, y con toda razón, que Günther Handl tilda al Trail Smelter como el locus classicus. ${ }^{40}$

Pues bien, del primero de los principios señalados, el párrafo que lo contiene y que es por mucho el más citado del laudo, es el siguiente:

Con arreglo a los principios de derecho internacional, así como el derecho de los Estados Unidos, ningún Estado tiene derecho a usar su territorio, o permitir el uso del mismo, en forma tal que se causen daños derivados de las emisiones en o hacia el territorio de otro Estado o las propiedades o personas ubicadas en él, cuando se produzca una consecuencia seria y que el daño se haya establecido con una evidencia clara y convincente. ${ }^{41}$

Esta afirmación, que no es otra cosa más que la traslación al derecho internacional de la máxima de derecho romano sic utere tuo ut alilenum non laedas (utiliza lo tuyo sin perjudicar a lo del otro), sería confirmada por la jurisprudencia internacional en el caso del Canal de Corfú, que si bien no se trata de un litigio ambiental, destaca porque en él la CIJ advirtió que existía un principio general bien reconocido sobre la obligación de los Estados de no permitir el uso de su territorio para actos contrarios a los derechos de otras naciones. ${ }^{42}$ No resulta extraño, por tanto, que un experto en materia ambiental internacional como Phillipe Sands entrelace los casos de Trail Smelter, Canal de Corfú y Lac Lanoux — que reseñaremos más adelante- como bases para el desarrollo del principio de buena vecindad, toral para el derecho ambiental internacional. ${ }^{43}$

40 Handl, Günther, "Territorial Sovereignty and the Problem of Transnational Pollution”, American Journal of International Law, vol. 69, núm. 1, 1975, pp. 50-76.

41 "Under principles of international law, as well as of the law of the United States, no State has the right to use or permit the use of its territory in such a manner as to cause injury by fumes in or to the territory of another or the properties or persons therein, when the case is of serious consequence and the injury is established by clear and convincing evidence".

42 ICJ, Corfu Channel Case (United Kingdom v. Albania), Merits, Judgmet of April 9th, 1949, p. 22.

43 Sands, Philippe, Principles of International Environmental Law, 3a. ed., Cambridge University Press, 2012, p. 197. 
Pues bien, la enunciación del laudo arbitral y su evolución daría pie a que la comunidad internacional desarrollara normas relativas a la responsabilidad por daños transfronterizos, como puede observarse en el Principio $21^{44}$ de la Declaración de la Conferencia de las Naciones Unidas sobre el Medio Humano (Estocolmo) de 1972 y que se tornaría en el Principio 2 de la Declaración de Rio:

De conformidad con la Carta de las Naciones Unidas y los principios del derecho internacional, los Estados tienen el derecho soberano de aprovechar sus propios recursos según sus propias políticas ambientales y de desarrollo, y la responsabilidad de velar por que las actividades realizadas dentro de su jurisdicción o bajo su control no causen daños al medio ambiente de otros Estados o de zonas que estén fuera de los límites de la jurisdicción nacional.

Debe destacarse que, a diferencia del laudo de la Trail Smelter Arbitration, el principio transcrito no requiere el estándar formulado en el arbitraje de una "evidencia clara y convincente" del daño, ni tampoco que éste sea calificado como una "consecuencia seria". Que el tribunal haya formulado estas exigencias pareciera haber sido orientado a evitar reclamaciones futuras a sabiendas que las vecindades producen inconveniencias naturales entre los Estados colindantes y, por otro lado, a que la prohibición del daño tendría dos caras: por un lado protegería a los granjeros estadounidenses y, por el otro, afectaría la producción de la fundidora con las consecuentes afectaciones económicas y sociales para ambos bandos. ${ }^{45}$

Retomemos el hilo, debe señalarse que el principio al que aludimos fue referenciado por la CIJ, sin las señaladas exigencias, tanto en la Opinión Consultiva sobre la Legalidad del Uso de Armas Nucleares como en el caso Gabčrkovo-Nagymaros, en los que apuntó el tribunal:

44 Literalmente señala el principio aludido: "De conformidad con la Carta de las Naciones Unidas y con los principios del derecho internacional, los Estados tienen el derecho soberano de explotar sus propios recursos en aplicación de su propia política ambiental y la obligación de asegurar que las actividades que se lleven a cabo dentro de su jurisdicción o bajo su control no perjudiquen al medio de otros Estados o zonas situadas fuera de toda jurisdicción nacional".

45 McCaffrey, Stephen C., "Of Paradoxes, Precedents, and Progeny: The Trail Smelter Arbitration 65 Years Later”, en Bratspies, Rebecca M. y Miller, Russel (eds.), Transboundary Harm in International Law Lessons from the Trail Smelter Arbitration, Cambridge University Press, 2006, pp. 37-40. 
La existencia de una obligación general de los Estados de asegurarse que las actividades que se realizan bajo su jurisdicción y control respeten el medio ambiente de otros Estados o de las áreas más allá de los límites de control nacional forma parte ahora del corpus del derecho internacional relacionado con el medio ambiente. $^{46}$

Además de haber refrendado la CIJ este punto en Construction of a Road in Costa Rica along the San Juan River (Nicaragua vs. Costa Rica), ${ }^{47}$ en otro ámbito su esencia se ha propagado en el articulado de otros instrumentos internacionales de la mayor relevancia. Entre ellos, el artículo 31 de la Convención sobre Diversidad Biológica de 1992, el Principio 1(a) de la Declaración de Principios Relativos a los Bosques, la Convención de las Naciones Unidas para la Lucha contra la Desertificación (preámbulo) y la Convención Marco de Naciones Unidas sobre el Cambio Climático (preámbulo).

En la Convemar el artículo 193 también se inspira en esta idea, siguiendo este lenguaje: "Los Estados tienen el derecho soberano de explotar sus recursos naturales con arreglo a su política en materia de medio ambiente y de conformidad con su obligación de proteger y preservar el medio marino", aunque puede argumentarse que el medio ambiente y el medio marino tendrían alcances más globales.

Lo mismo puede señalarse del preámbulo propuesto por la Comisión de Derecho Internacional (CDI) dentro del tópico Prevención de daños transfronterizos causados por actividades riesgosas, cuyo numeral tercero, bajo el simple título de prevención, señala: "El Estado de origen deberá tomar todas las medidas necesarias para prevenir un daño transfronterizo significativo y, en cualquier caso, minimizar el riesgo a ello" . ${ }^{48}$ Léase que la CDI añade "significativo" a la enunciación llana que se había encontrado en otros instrumentos.

46 "The existence of the general obligation of States to ensure that activities within their jurisdiction and control respect the environment of other States or of areas beyond national control is now part of the corpus of international law relating to the environment". Cfr. Nuclear Weapons, 1996, I. C. J. 226, at 241-42, para. 29 (July 1996). 48 Gab̌r 1 kovo-Nagymaros Project (Hun./Slov.), 1997, I. C. J. 7, at 41, para. 53 (Sept. 25).

47 Construction of a Road in Costa Rica along the San Juan River (Nicaragua v. Costa Rica), I. C. J., para 118, Judgment of 16 December 2015.

48 "The State of origin shall take all appropriate measures to prevent significant transboundary harm or at any event to minimize the risk thereof". Anuario de la Comisión de Derecho Internacional, 2004, vol. III, segunda parte, capítulo VII, pp. 67-98. 
La misma influencia de la esencia del laudo puede observarse en la formulación del artículo 7o. de la Convención sobre el Derecho de los Usos de los Cursos de Agua Internacionales para Fines Distintos de la Navegación, ${ }^{49}$ basado en el trabajo de la CDI en el mismo tema: "Los Estados en el curso de agua, al utilizar un curso de agua internacional en sus territorios, adoptarán todas las medidas apropiadas para impedir que se causen daños sensibles a otros Estados del curso de agua". En este punto debemos hacer notar que, a diferencia de los otros instrumentos señalados, esta convención añade a la fórmula un ingrediente: los daños deben de ser "sensibles", cuestión que resulta subjetiva y que podría elevar el estándar en cuanto a la valoración de los daños.

El número de documentos inspirados en el fallo arbitral podría incrementarse, algunos de ellos de gran importancia para el derecho ambiental internacional, como el Reporte de la Brundtland Commission. Del acopio de la multitud de instrumentos que se inspiran en el fallo de la Trail Smelter Arbitration puede concluirse que el principio de usar el territorio sin causar daños ambientales a otros Estados ya es un principio consagrado en el derecho internacional.

Por lo que toca al segundo de estos principios, "el que contamina paga", el tribunal tuvo la primicia de aplicar esta fórmula en la esfera internacional. ${ }^{50}$ De una manera simplista, podría resumirse que el tribunal arbitral trasladó el principio en el caso sometido en dos puntos: i) la obligación del pago monetario, y ii) la previsión de mecanismos para evitar la emisión de químicos dañinos de la fundidora. Irónicamente, en su tiempo el fallo fue apreciado como un triunfo de la fundidora; ${ }^{51}$ la teoría de los daños "invisibles”, orientados a la tierra y que había avanzado la parte estadounidense, fue desechada. Recordemos que el estándar fijado era alto tanto en la parte procesal, que requería una "evidencia clara y convincente" del daño, como en la parte sustantiva, que apunta a que la actividad debía producir una "consecuencia seria”.

49 A/RES/51/229, adoptada el 21 de mayo de 1997.

50 Ellis, Jaye, "Has International Law Outgrown Trail Smelter?", en Bratspies, Rebecca y Miller, Russell (eds.), Transboundary Harm in International Law: Lessons from the Trail Smelter Arbitration, Cambridge University Press, 2006, p. 133.

51 Los granjeros estadounidenses sólo pudieron incrementar $\$ 78,000$ USD a los $\$ 350,000$ originalmente concedidos por la Comisión Internacional Conjunta. 
El estudioso del derecho ambiental internacional reconocerá que es incipiente la configuración del principio de "el que contamina paga" en este litigio. Bien es cierto, como apunta Alonso Gómez Robledo, que el arbitraje no establece un régimen de responsabilidad objetiva, cuestión que no fue sometida al Tribunal por haber sido aceptada tácitamente por Canadá al someterse al arbitraje. ${ }^{52} \mathrm{~A}$ pesar de ello, después del arbitraje del Trail Smelter quedó impreso en los anales jurisprudenciales internacionales que una reclamación ambiental podría ser adjudicada y que habría responsabilidad en los casos de daños ecológicos transfronterizos.

\section{ARbitRaje DEL LAC LANOUX ENTRE FRANCIA Y ESPAÑA}

\section{Antecedentes}

El lago de Lanós, lac Lanoux en francés y estany de Lanós en catalán, está ubicado en Francia, en la vertiente sur de los Pirineos. Este lago, nutrido por corrientes francesas, alimenta, a su vez, al río Carol que, después de recorrer 25 kilómetros en territorio francés, atraviesa la frontera española y discurre alrededor de seis kilómetros en el territorio de España, antes de unirse al río Segre para desembocar finalmente en el río Ebro.

Puede, en muy resumidas cuentas, señalarse que la controversia del lago Lanoux surgió ante la pretensión española de imponer a Francia la obligación de llegar a un acuerdo antes de explotar las capacidades eléctricas del flujo de la corriente producida por el Lanoux. El aprovechamiento de las aguas del Lanoux fue objeto de consultas entre ambos gobiernos entre 1917 y 1956. En 1952, la Electricité de France presentó un proyecto de desviación de las aguas del lago Lanoux hacia el río Ariège para la producción de energía eléctrica y la plena restitución del flujo a través una galería subterránea hacia el Carol. Además del mecanismo de compensación, Francia había ofrecido garantías de orden técnico, entre ellas la creación de una comisión mixta. A pesar de ello, España temía

52 Gómez-Robledo Verduzco, Alonso, Responsabilidad internacional por daños transfronterizos, 2a. ed., México, UNAM, Instituto de Investigaciones Jurídicas, 2014, p. 36. 
que se lesionaran sus derechos y que la obra sería contraria al tratado de límites firmado en Bayona el 26 de mayo de 1866 y al acta adicional de la misma fecha entre Francia y España.

Dicho sea de paso, la actitud francesa dista mucho de la funesta Doctrina Harmon, la cual sostenía que aquel país podía hacer lo que quisiera con los cursos de agua en su territorio sin importar el daño que causara a los Estados vecinos, postura sustentada en 1895 por el procurador general Harmon de EUA al ser consultado sobre los perjuicios sufridos por agricultores mexicanos después de haberse desviado el curso del río Bravo para favorecer la irrigación del lado estadounidense. No cabe duda que la buena vecindad tiene una doble cara, al revertirse la propia doctrina después de haberla padecido ante Canadá por el uso del río Columbia. ${ }^{53}$

Regresando al arbitraje, España argumentó que el tratado y acta mencionados disponían que las obras no podían emprenderse sin un acuerdo previo entre ambos países, mientras que Francia opinaba que actuaba legalmente sin tal acuerdo, y que el tribunal arbitral tendría que decidir de acuerdo al compromiso arbitral ${ }^{54}$ firmado por ambos países el 19 de noviembre de $1956 .{ }^{55}$

\section{Actuación del tribunal}

El 6 de noviembre de 1957 el tribunal arbitral resolvió a favor de Francia por mayoría de cuatro de sus cinco miembros, ${ }^{56}$ señalando que los

53 Para un estudio a fondo de esa doctrina véase Cruz Miramontes, Rodolfo, "La doctrina Harmon, el Tratado de Aguas de 1944 y algunos problemas derivados de su aplicación”, Foro Internacional, vol. 6, núm. 1 (21), 1965, pp. 12-49.

54 Así lo prevendría el artículo 1o.: "Le Tribunal sera prié de répondre à la question suivante: Le Gouvernement français est-il fondé à soutenir qu'en exécutant, sans un accord préalable entre les deux Gouvernements, des travaux d'utilisation des eaux du lac Lanoux dans les conditions prévues au projet et aux propositions français visés au préambule du présent compromis, il ne commettrait pas une infraction aux dispositions du Traité de Bayonne du 26 mai 1866 et de l'Acte additionnel de la même date?".

55 Desde el 10 de julio de 1929 ambos países habían firmado una convención mediante la cual quedarían obligadas al arbitraje.

56 De los dos árbitros a los que tuvo derecho a seleccionar España, solamente uno de ellos tuvo una opinión disidente. 
trabajos emprendidos eran compatibles con el Tratado de Bayona y el acta adicional..$^{57}$

El laudo arbitral tenía como limitación la aplicación del derecho conforme los dos instrumentos convencionales. ${ }^{58}$ Sobre este punto, el propio laudo recordó que la Corte Permanente de Justicia Internacional así había limitado su propia competencia en un caso similar en una disputa por el uso entre Bélgica y los Países Bajos por el río Meuse, en el que encontró que ninguno de esos países había violado sus obligaciones derivadas del tratado bilateral que regulaba el aprovechamiento de ese río por la construcción de canales, esclusas y represas. Ello no obstó para que el tribunal apreciara sus conclusiones conforme al derecho internacional aplicable al caso para ayudar a la interpretación. Debe señalarse que la decisión reafirmó, desde el primer punto, el principio de soberanía territorial $;^{59}$ es decir, que cada Estado ejerce pleno y exclusivo imperio sobre su territorio con las limitaciones impuestas por el derecho internacional. ${ }^{60}$ Asimismo, el laudo en el Affaire du Lac Lanoux ratificó que la buena fe se presume, ${ }^{61}$ lo que conlleva que la carga de la prueba recaiga en aquel que la reclama.

El tribunal ahondó en el primer punto, señalando que las restricciones a la soberanía deben de ser evidentes. Particularmente, el tribunal razonó que admitir la necesidad de un acuerdo previo sería "admitir un «derecho de veto» que paralizaría, a discreción de un Estado, el ejercicio de la competencia territorial de otro Estado" (punto 11).

Debe tenerse cuidado en el contexto, el tribunal había observado que la actuación de Francia no provocaba perjuicios en el lado español. Por otro

57 Affaire du lac Lanoux (Espagne, France), Recueil des Sentences Arbitral, 16 November 1957, vol. XII, pp. 281-317, reproducido también en Revue générale de droit international public, t. LXII, 1958, p. 79.

58 Diversion of Water from the Meuse, Judgment of 28 June 1937, PCIJ (1937), Ser. A/B, núm. 70, p. 450.

59 El tribunal ya tenía el precedente judicial sobre el ejercicio de la soberanía asentado por la CPJI en el Lotus Case.

60 Desde el primer punto el laudo señala: "La soberanía territorial surte el efecto de una presunción. Debe ceder ante todas las obligaciones internacionales, sea cual fuere el origen de éstas, pero no cede sino ante éstas".

61 El punto 8 del laudo afirma: "hay un principio general de derecho bien establecido según el cual la mala fe no se presume”, cuestión que ya tenía un antecedente judicial en la Corte Permanente de Justicia Internacional, véase Case concerning certain German Interests in Polish Upper Silesia (Germany v. Poland) (Merits), 1926, PCIJ Series A, no. 7, 25th May 1926, 30. 
lado, la colocación temporal del arbitraje está ubicada en una época en la que el aprovechamiento de la naturaleza se veía como de servicio para el bien de todos y que el riesgo del uso abusivo no había conducido a que se tuviera que someter la detentación de los medios de acción a otros Estados. ${ }^{62}$ He aquí, por lo menos en la última parte, la concepción que hoy puede ser primitiva para nociones más modernas, como el desarrollo sustentable.

Queda algo lejana también la decisión del Tribunal a la aspiración actual de que los Estados lleven a cabo evaluación del impacto ambiental (Principio 17) y, en particular, al Principio 19 de la Declaración de Río, que señala:

Los Estados deberán proporcionar la información pertinente y notificar previamente y en forma oportuna a los Estados que posiblemente resulten afectados por actividades que puedan tener considerables efectos ambientales transfronterizos adversos, y deberán celebrar consultas con esos Estados en una fecha temprana y de buena fe.

Podría, bien es cierto, discutirse que la obligación de informar a los Estados es norma emergente que aparece cada vez más en la práctica de los propios Estados. ${ }^{63}$ Sin embargo, también debe reconocerse que la propia CDI señaló que "El principio de la buena fe es parte integrante de toda obligación de consulta y negociación. La obligación de consultar y negociar sinceramente y de buena fe se reconoció en el laudo del Affaire du Lac Lanoux" ${ }^{64}$ Para llegar a esta conclusión la CDI retomó del texto del laudo: "Las consultas y negociaciones entre los dos Estados deben ser sinceras, deben cumplir las normas de la buena fe y no deben ser simples formalidades. Las normas de

62 Literalmente señala, en el punto 9: "El dominio creciente del hombre sobre las fuerzas y los secretos de la naturaleza, ha puesto en sus manos instrumentos de los cuales puede servirse, tanto para violar sus obligaciones, como para el bien común de todos; el riesgo de empleo abusivo no ha conducido, hasta el presente, a someter la detentación de estos medios de acción a la autorización de los Estados eventualmente amenazados”.

63 El deber de informar se encuentra en el principio 19 de la Declaración de Río, así como en un buen número de instrumentos internacionales y práctica de los Estados. Véase Kiss, Alexander y Shelton, Dinah, Guide to International Environmental Law, Leiden/Boston, Brill, 2007, pp. 100-102.

64 "Responsabilidad internacional por las consecuencias perjudiciales de actos no prohibidos por el derecho internacional". Comisión de Derecho Internacional, Yearbook of the International Law Commission, vol. II, part two, A/CN.4/SER.A/2001/Add.1 (part 2), p. 171. 
la razón y la buena fe son aplicables a los derechos y obligaciones de procedimiento relativos al uso compartido de los ríos internacionales".

En efecto, el laudo señala que el Estado de aguas arriba tiene un derecho de iniciativa, aunque si durante el curso de las conversaciones el Estado de aguas abajo le somete proyectos debe examinarlos, reteniendo el derecho de preferir la solución adoptada por su proyecto (punto 23). Este razonamiento es el comienzo de una cautela de los intereses del Estado de aguas abajo, que con claridad expresó el Tribunal: "Hay que tener en cuenta, sea cual fuere su naturaleza, todos los intereses que podrían ser afectados por los trabajos emprendidos, incluso si no corresponden a un derecho", y abundaría:

El Tribunal opina que el Estado de aguas arriba tiene, según las reglas de la buena fe, la obligación de tomar en consideración los intereses en presencia, de buscar la forma de dar todas las satisfacciones compatibles con la consecución de sus propios intereses y de mostrar que tiene a este respecto un deseo real de conciliar los intereses del otro ribereño con los suyos propios.

ElTribunal no tenía más que reconocer que el artículo 11 del acta adicional de 1866 establecía entre las partes un deber de notificación, así como la obligación del establecimiento de un régimen de reclamaciones y de salvaguarda de los intereses de las partes. Aparejada a la obligación de notificación, el Tribunal indicó que si no se ha efectuado el aviso, que al otro Estado ribereño le asiste el derecho a "exigir notificación de los trabajos o concesiones que constituyen el objeto de un proyecto” (punto 21). No obstante, también el Tribunal dejó claro que la notificación no crea la obligación de instituir un previo acuerdo para comenzar con las actividades (punto 16) ${ }^{65}$

El tribunal reconoció la obligatoriedad y el contenido de las consultas, el texto detallaría que "habría obligación de aceptar de buena fe todas aquellas conversaciones y contactos susceptibles mediante una amplia confrontación de intereses y una recíproca buena voluntad, de colocarlos en las mejores condiciones para hacer posible la estipulación de tales acuerdos" (punto 13). Más adelante indicaría que para apreciar el modo

65 En sus propios términos, el laudo señala en la parte conducente: "la obligación de la previa notificación no implica la otra, mucho más amplia, de obtener el acuerdo del Estado notificado". 
en que un proyecto toma en cuenta los intereses de las partes debe encontrarse en las negociaciones "el inventario de los intereses que han podido alegarse, el precio que cada una de las Partes está dispuesta a pagar para obtener su salvaguardia".

En resumen, se desprende entonces que las consultas y negociaciones deben ser de buena fe, tomando en cuenta los intereses de los Estados que puedan resultar afectados. Asimismo, se prevé la posibilidad de que, pese a todo, las partes no lleguen a un acuerdo, lo que no imposibilita que el Estado del proyecto continúe con su actividad. En ese supuesto, el que realiza el proyecto continúa obligado a tener en cuenta los intereses que pudieran afectarse de los otros Estados, ${ }^{66}$ lo cual se desarrolló en el apartado anterior sobre el caso Trail Smelter, por lo que debe entenderse que en el supuesto de perjudicar al Estado de aguas abajo podría incurrir en responsabilidad.

Puede encontrarse en estos puntos un cierto paralelismo entre el caso de las papeleras sobre el río Uruguay de la $\mathrm{CIJ} .{ }^{67} \mathrm{En}$ ambos casos se circunscribió a la aplicación de instrumentos bilaterales, se constató la existencia de las obligaciones de notificación, consultas y negociaciones, y la violación de este deber no implicó la suspensión de las actividades reclamadas.

\section{LA HERENCIA DE LOS PRIMEROS ARBITRAJES}

Los primeros arbitrajes internacionales en materia ambiental sentaron principios fundamentales para el derecho ambiental internacional. Aunque el primero de ellos no haya tenido la resonancia de los subsecuentes arbitrajes en términos de citas posteriores en jurisprudencia, el Fur Seals Arbitration dejó en claro que un Estado puede ejercer medidas de control sobre sus propios recursos naturales en su territorio, y que, con el concurso de Estados involucrados, es posible alcanzar un acuerdo para la conservación de especies a través de instrumentos internacionales.

66 Eso fue tomado en cuenta por la CDI para la elaboración del tercer párrafo del artículo 9o., "Consultas sobre las medidas preventivas", del texto del proyecto de artículos sobre prevención del daño transfronterizo resultante de actividades peligrosas.

67 ICJ, Case Concerning Pulp Mills on the River Uruguay, Argentina v. Uruguay, Judgment on the Merits, ICGJ 425 (ICJ 2010), 20th April 2010. 
Ahora bien, el recurso al derecho natural, como lo empleó Carter, parece tener eco en la jurisprudencia internacional y en la doctrina a cargo del juez Cançado Trindade, lo cual resulta interesante ante los huecos y la falta de compromisos sólidos en algunas materias importantes de derecho ambiental. Este recurso no es desdeñable, tomando en cuenta que los avances del deterioro a nuestro entorno son más acelerados que la respuesta de los Estados.

Por otro lado, la decisión en el Trail Smelter Arbitration es precursora de los principios de prevención de daño y "el que contamina paga", conceptualizaciones que han evolucionado y se han extendido en el derecho ambiental internacional. Por su parte, el laudo en el Affaire du Lac Lanoux es un antecedente importante para las obligaciones de notificaciones previas, consultas y negociación. Puede enlazarse entre estos dos arbitrajes, como lo hace el profesor Aaron Schwabach, que el Affaire du Lac Lanoux "añadió un elemento de balance: los Estados tienen derecho a aprovechar sus recursos dentro de sus fronteras libre de toda interferencia exterior siempre y cuando que ese aprovechamiento no produzca un daño fuera de sus límites fronterizos". ${ }^{68}$

Mucho es el camino recorrido del principio de "el que contamina paga" desde su concreción en el Trail Smelter Arbitration hasta nuestros días. Es, sin embargo, un antecedente para la definición de la Organización para la Cooperación y el Desarrollo Económicos (OCDE) ${ }^{69}$ y la enunciación del Principio 16 de la Declaración de Río ${ }^{70}$ en cuanto a la atribución del de-

68 "[T] $[$ he Lac Lanoux decision added a balancing element: States had a right to develop resources within their own borders free from outside interference, so long as that development resulted in no harm beyond the state's borders". Cfr. Schwabach, Aaron, International Environmental Disputes: A Reference Handbook, California, ABC-CLIO, 2011.

69 OCDE, Recomendation of the Council on Guiding Principles Concerning International Economic Aspects of Environmental Policies, 26 de mayo de 1972, disponible en: https://legalinstruments. oecd.org/en/instruments/OECD-LEGAL-0102.

70 Literalmente señala: "PRINCIPIO 16. Las autoridades nacionales deberían procurar fomentar la internalización de los costos ambientales y el uso de instrumentos económicos, teniendo en cuenta el criterio de que el que contamina debe, en PRINCIPIO, cargar con los costos de la contaminación, teniendo debidamente en cuenta el interés público y sin distorsionar el comercio ni las inversiones internacionales”. Para el caso de México, en el sentido directo de atribución de la responsabilidad y la consecuente reparación, el avance es claro con la introducción de las acciones colectivas en materia ambiental conforme a la Ley de Responsabilidad Ambiental que entró en vigor en 2013. En el caso europeo, se resume en la 
ber de reparación del contaminador una vez producido el daño ambiental. Debe señalarse que el principio de "el que contamina paga" tiene mayores connotaciones y puede explicar la deuda ecológica histórica de los países del norte a los países del sur que aborda el Protocolo de Kioto, cuestión que va más allá de este estudio.

En otro punto, no debe dejarse de lado la contribución de las decisiones en el Trail Smelter Arbitration y el Affaire du Lac Lanoux para el régimen de la responsabilidad internacional por daños transfronterizos, lo que ha sido reconocido por la CDI en el estudio sobre la "Responsabilidad internacional por las consecuencias perjudiciales de actos no prohibidos por el derecho internacional”.

Asimismo, el régimen de recursos hídricos internacionales ha alcanzado una evolución importante en la concepción de normas, aunque se ha batallado enormemente para que el instrumento que lo regula tenga alcances amplios. El 17 de agosto del 2014 entró en vigor la Convención sobre el Derecho de los Usos de los Cursos de Agua Internacionales para Fines Distintos de la Navegación, ${ }^{71}$ adoptada por la Asamblea General de las Naciones Unidas en 1997, la cual sólo ha sido firmada por Venezuela y Paraguay en el ámbito latinoamericano. Cabe señalar que dicho instrumento recoge el principio de prevención de daños ambientales recogido en el Trail Smelter Arbitration y que también puede desprenderse del arbitraje sobre el Affaire du Lac Lanoux. Literalmente, el artículo 7o. del citado instrumento señala:

1. Los Estados del curso de agua, al utilizar un curso de agua internacional en sus territorios, adoptarán todas las medidas apropiadas para impedir que se causen daños sensibles a otros Estados del curso de agua.

2. Cuando a pesar de ello se causen daños sensibles a otro Estado del curso de agua, el Estado cuyo uso los cause deberá, a falta de acuerdo con respecto a ese uso, adoptar todas las medidas apropiadas, teniendo debidamente en cuenta lo dispuesto en los artículos 5o. y 6o. y en consulta con el Estado afectado, para eliminar o mitigar esos daños y, cuando proceda, examinar la cuestión de la indemnización.

Directiva 2004/35/CE, sobre responsabilidad medioambiental en relación con la prevención y reparación de daños medioambientales.

71 Al 6 de marzo de 2020, solamente 36 Estados son parte. 
Es de esta manera como los tres arbitrajes reseñados iniciaron con la cimentación de los primeros principios en materia ambiental, abriendo el camino a la formulación de nuevos principios que dan forma a la evolución del derecho ambiental internacional, materia en la que falta un largo trecho que recorrer para alcanzar la aspiración de "satisfacer las necesidades de las generaciones presentes sin comprometer las posibilidades de las generaciones del futuro para atender sus propias necesidades", como fue definido el desarrollo sostenible en la Cumbre de Johannesburgo (2002). No cabe duda que la jurisprudencia internacional es una puerta para la consolidación de los principios en materia ambiental.

VII. BIBLIOGRAFÍA

Monografías

AQuino, Tomás, Suma de Teología, parte II-II (a), trad. de Lorenzo Jiménez Patón, Madrid, Biblioteca de Autores Cristianos, 1990, edición bilingüe en 16 tomos.

BIRNIE, Patricia et. al., International Law and the Environment, 3a. ed., Hampshire, Oxford University Press, 2009.

BODANSKY, Daniel, The Art and Craft of International Environmental Law, Cambridge, Harvard University Press, 2010.

BRAtSPIES, Rebecca y MilleR, Russell (eds.), Transboundary Harm in International Law: Lessons from the Trail Smelter Arbitration, Cambridge University Press, 2006.

BROWn WeISS, Edith, In Fairness to Future Generations. International Law, Common Patrimony and Intergenerational Equity, Tokio, United Nations University Press, 1989.

FERRER ORTEGA, Luis Gabriel, Los derechos de las futuras generaciones desde la perspectiva del derecho internacional: el principio de equidad intergeneracional, México, UNAM, Instituto de Investigaciones Jurídicas, 2014, disponible en: https://biblio.juridicas. unam. $\mathrm{mx} / \mathrm{bjv} /$ detalle-libro/3635-losderechos-de-las-futuras-generaciones-desde-la-perspectiva-del-derecho-inter nacional-el-principio-de-equidad-intergeneracional. 
Fitzmaurice, Malgosia, Contemporary Issues in International Environmental Law, Cheltenham, Edward Elgar Publishing, 2009.

GÓmeZ-Robledo VerduzCO, Alonso, Responsabilidad internacional por daños transfronterizos, 2a. ed., México, UNAM, Instituto de Investigaciones Jurídicas, 2014.

KISS, Alexander, "Problèmes juridiques de la pollution de l'air”, Recueil des Cours de la Academie de Droit International, 1973.

Kiss, Alexander y Shelton, Dinah, Guide to International Environmental Law, Leiden/Boston, Brill, 2007.

LOCKE, John, Two Treatises of Government, Londres, A. Millar et al., 1764, disponible en: https://oll.libertyfund.org/titles/locke-the-two-treatises-ofcivil-government-hollis-ed.

LOUKA, Elli, International Environmental Law: Fairness, Effectiveness, and World Order, Cambridge University Press, 2006.

LYSTER, Simon, International Wildlife Law: An Analysis of International Treaties Concerned with the Conservation of Wildlife, Cambridge University Press, 1985.

NANDA, Ved P., International Environmental Law \& Policy for the 21 st Century, 2a. ed., Leiden, Martinus Nijhoff Publishers, 2013.

NeWTOn E., David, Environmental Justice: A Reference Handbook, 2a. ed., California, ABC-CLIO, LLC, 2009.

Older, Owen S., Conservación de recursos naturales, 2a. ed., trad. de Ambrosio González Cortés, México, Pax, 2000.

PufFEndorf, Samuel, Of the Law of Nature and Nations, Londres, J. Walthoe et al., 1729, disponible en: https: / / openlibrary.org/books/OL6563001M/ Of_the_law_of_nature_and_nations.

SANDS, Philippe, Principles of International Environmental Law, 3a. ed., Cambridge University Press, 2012.

SCHWABACH, Aaron, International Environmental Disputes: A Reference Handbook, California, ABC-CLIO, 2011.

STEPHENS, Tim, International Courts and Environmental Protection, Cambridge, Cambridge Studies in International and Comparative Law, 2009.

Tremmel, Jörg, A Theory of Intergenerational Justice, Londres, Earthscan, 2009.

United Nations Environment Programme, Compendium of Judicial Decisions in Matters Related to Environment, International Decisions, Nairobi, United Nations Environment Programme, 1998. 
Esta revista forma parte del acervo de la Biblioteca Jurídica Virtual del Instituto de Investigaciones Jurídicas de la UNAM

\section{Artículos}

BenadAVA, Santiago, "Las cuestiones de interés general en el caso del lago Lanoux”, Revista de Derecho Público, núm. 5/6, 1966.

Brown, J. Stanley, "Fur Seals and the Bering Sea Arbitration", Journal of the American Geographical Society of New York, vol. 26, núm. 1, 1894.

BROWN WEISS, Edith, “The Evolution of International Environmental Law”, 54 JapaneseY. B. Intl. L.

CASTILlO Daudí, M., "La protección y preservación de cursos de agua internacionales: el Convenio sobre el derecho de los usos de los cursos de agua internacionales para fines distintos de la navegación de 21 de mayo de 1997”, Anuario Español de Derecho Internacional, vol. XV, 1999.

CRuz Miramontes, Rodolfo, "La doctrina Harmon, el Tratado de Aguas de 1944 y algunos problemas derivados de su aplicación", Foro Internacional, vol. 6, núm. 1 (21), 1965.

DECLEVA, M., “Sentenza arbitrale del 16-IX-1957 nell' affare dell'utilizzazione delle acque del lago Lanoux", Diritto Internazionale, vol. XIII.

DULÉRY, F. "'L’Affaire du lac Lanoux", Revue Générale de Droit International Public, t. LXII, 1958.

Ferrer Ortega, Luis Gabriel y Ferrer OrtegA, Jesús Guillermo, "El problema de la fundamentación filosófica de los derechos de las generaciones futuras", Anuario Mexicano de Derecho Internacional, México, vol. VIII, 2008.

GERVAIS, André, "La sentence arbitrale du 16 novembre 1957 réglant le litige franco-espagnol relatif à l'utilisation des eaux du Lac Lanoux", Annuaire Français de Droit International, 1957.

HANDL, Günther, "Territorial Sovereignty and the Problem of Transnational Pollution”, American Journal of International Law, vol. 69, núm. 1, 1975.

JENnings, (Sir) Robert Y., "Judicial Reasoning at an International Court", en RESS, G. (ed.), Vorträge Reden und Berichte aus dem Europa-Institut, Saarbrücken, núm. 236, 1991.

JIMÉNEZ DE ARECHAGA, Eduardo, "Normas jurídicas internacionales que regulan el aprovechamiento hidráulico", Inter-Am. L. Rev., 2, 1960.

LAYLiN, John G. y BiANCHI, Rinaldo L., "The Role of Adjudication in International River Disputes: The Lake Lanoux Case", The American Journal of International Law, 53.1, 1959.

Mestre, A., "Quelques remarques sur l'Affaire du lac Lanoux", Mélanges Offerts à Jacques Maury, París, Dalloz, 1960. 